P9 (continued)

U.S. children aged 2-5 consume at least 1 SSB daily, with considerable variation by demographics.

Objective: To explore SSB-related beliefs and practices among a sample of low-income, urban parents of young children.

Study Design, Setting, Participants: Fifteen English language interviews were conducted with parents of children aged 2-5 at a family health clinic in Peekskill, New York. Interviews were recorded, transcribed, and analyzed for major themes using standard qualitative methods.

Measurable Outcome/Analysis: Several themes emerged: few parents expressed clear knowledge of or had discussed SSB with their child's health care provider; participants who could not explain the difference between $100 \%$ juice and fruit drinks tended to serve SSB more frequently and in higher quantities; nearly all participants believed that products labeled "natural" or "all natural" were healthful; most parents named low cost and children's preferences/nagging as reasons they serve SSB; and WIC participants believed juice was healthful because it can be purchased with vouchers.

Results: Parents who serve SSB may lack consistent, reliable information from physicians and health authorities. They may be misled by labels and influenced by cost and pressure from their children.

Conclusions: Lowering SSB consumption has the potential to reduce calorie intake, improve diet quality, and reduce obesity risk in young children. Consistently employing recommended SSB screening protocols during well-child visits is one practical preventive approach. Changes to product labeling, improving public education about and taxing SSB may also help reduce intake.

Funding: None.

\section{P10 Blended Learning: Use of Instructional Videos in an Undergraduate Food Preparation Lab}

Georgia Jones,PhD, gjones2@unl.edu, Nebraska Extension, 119D Ruth Leverton Hall, Lincoln, NE, 68583; Lauren Rathman, MS, Grand Island Public Schools

Objective: The goal of this research study was to determine if supplemental, instructional videos would be beneficial in teaching scientific concepts and preparation procedures in a sophomore introductory foods class.

Use of Theory or Research: Students no longer enter college with basic food knowledge and are therefore not prepared to understand the science of food. Observation from instructors and researchers support this. Knowledge of food is essential in preparing nutrition and dietetic students to be competent in their careers.

Target Audience: College students enrolled in an introductory foods class.

Course/Curriculum Description: Scientific Principles of Food Preparation is taught at a 4-year university. It consists of a 1-hour laboratory and a 3-hr lecture. Fifteen instructional videos focusing on the science and preparation of food were developed to help students build foundational knowledge. The videos were no more than 4 minutes in length. This study consisted of 2 classes. One class served as the experimental group, while the other served as the control. The experimental group viewed the videos before conducting experiments, completing prelab quizzes, and lab reports. The control group viewed the videos after conducting experiments, completing pre-lab quizzes, and lab reports. There were 15 students in each group.

Evaluation Methods: Students' pre-lab quizzes and lab reports were graded as usual. The averages for the pre-lab quizzes, lab reports, and the final grades were compared. Data was analyzed using Analysis of Variance (ANOVA).

Results: There was a significant difference $(P<.05)$ in the pre-lab quiz and lab report, and final class grades between the control and experimental group. The experimental group scored significantly higher on all measures.

Conclusions: As evidenced by the grades for both groups of students, the ability to view the videos before the start of lab did improve learning. Students who read the lab manual and viewed the videos were exposed to the same material twice. Developing videos may be one way to help nutrition students understand scientific concepts of food preparation.

Funding: None.

\section{P11 Nutrition Insights on Dietary Preferences and Challenges From Lower SES Adults with Heart Failure}

Deborah Schnur, PhD, MPH, schn0447@umn.edu, University of Minnesota, Division of Epidemiology and Community Health, 1300 South Second St, Minneapolis, MN, 55454; Lisa Harnack, DPH, RD, University of Minnesota, Division of Epidemiology and Community Health

Background: Only a limited number of studies have investigated challenges heart failure (HF) patients face in adhering to dietary recommendations for HF management, and none have focused on racially diverse, lower socioeconomic status (SES) populations at high risk for adverse outcomes.

Objective: The objective of this study was to explore and understand the dietary preferences and challenges of older, lower SES adults with HF.

Study Design, Setting, Participants: We conducted an exploratory, qualitative study using structured telephone interviews. Convenience sampling was used to recruit 21 adults with $\mathrm{HF}$, aged 50 years or older, from the Minneapolis-St. Paul metropolitan area.

Measurable Outcome/Analysis: Responses to openended questions about participants' experiences managing their diet, grocery shopping, and cooking were analyzed from interview audio recordings using thematic analysis. Responses to closed-ended questions, including those about demographics and health and nutrition characteristics, were analyzed using descriptive statistics. 


\section{P11 (continued)}

Results: The dietary recommendations and goals most frequently mentioned by participants were limiting sodium and increasing intake of fruits and vegetables. Many participants reported that high food prices and a limited budget made it difficult to follow their diet and make healthy choices when grocery shopping. The food environment, particularly the high-salt content in food products and the promotion of unhealthy food in stores, presented additional challenges. Participants suggested a variety of ways to make following their diet, shopping, and cooking easier including online grocery ordering and delivery, pre-prepared ingredients, and assistance with meal planning, shopping, cooking, and cleaning up. The idea of a medically tailored home-delivered meal service was well-received by most study participants.

Conclusions: Our findings provide a starting point for developing programs and policies to help older, lower SES HF patients follow their diet and improve their health, thereby reducing health disparities. Since finances are a key concern, programs and policies need to be designed with cost in mind.

Funding: J.B. Hawley Research Award from the Division of Epidemiology and Community Health at the University of Minnesota

\section{P12 Cooking Self-Efficacy, Perceived Health Status, and Fresh Produce Purchasing Criteria Among Faculty and Staff}

Georgianna Mann, PhD, grmann@olemiss.edu, University of Mississippi, PO Box 1848, Sorority Row, Lenior 220, University, MS, 38677; Laurel Lambert, PhD, LD, RDN, University of Mississippi; Kritika Gupta, MS, University of Mississippi; Selby Greer, MS, University of Mississippi

Background: National health guidelines emphasize the nutritional benefits of fruit and vegetable (FV) consumption. Positive associations between FV consumption, health awareness, and cooking skills have been noted in previous work. However, little is known about this relationship among university faculty and staff.

Objective: To explore the relationship between cooking skills, health perceptions, importance of FV consumption, and FV purchase criteria among university faculty and staff by gender.

Study Design, Setting, Participants: A random representative sample of faculty and staff at a large Southern university were recruited to complete an online survey in spring $2018(\mathrm{n}=175)$.

Measurable Outcome/Analysis: Questions included cooking skills, perceived health status, benefits of produce consumption, produce purchasing criteria, and demographic data. Associations between cooking skills, selfhealth assessment, and fresh produce buying preferences were evaluated using Pearson's correlation test. Independent t-tests were used to determine differences by gender. Significance was determined by $P \leq .05$.

Results: Health perceptions were positively correlated with perceived health benefits of fresh produce $(P<.05)$,
non-GMO/Organic $(P<.05)$, and locally grown $(P<.05)$. Female participants reported higher cooking skill levels. Cooking skills were positively associated with grocery shopping responsibilities $(P<.01)$, shopping at the farmer's market $(P<.01)$, and home gardens $(P<.05)$.

Conclusions: In this study, females responded with the highest cooking skill levels which positively correlated with importance of fresh produce, specific purchasing preferences, and self-perceived health status. While overall cooking skill level in this sample was high, it is likely that those interested in healthy eating were more likely to complete the survey. Universities could play an important role in improving fresh produce consumption among faculty and staff by providing support and resources in the form of cooking classes through university programs such as nutrition departments, university wellness programs, and community venues.

Funding: None.

\section{P13 Social Network Analysis of Urban Church Food Pantries}

Tracy Noerper, PhD, LDN, RDN, Tracy.Noerper@Lipscomb. edu, Lipscomb University, One University Park Dr, Nashville, TN, 37204; Janet Colson, PhD, RDN; Andrew Owusu, PhD

Background: Examining social networks helps to explain the connectedness of individuals, groups or communities, and is useful in demonstrating the potential that lies within and between networks. When evaluating social networks, relationships are viewed as "nodes" (eg individuals or points) and their connecting "ties" (eg the relationships between the nodes). Detailing food pantry and client "connectedness" through social network analysis can assist researchers and policymakers to enhance efficiency and effectiveness of the informal emergency food system.

Objective: The objective of the study was to examine the degree to which urban church food pantries are connected to the clients they serve using the social network theory.

Study Design, Setting, Participants: The research design was a population-based, cross-sectional study using a snowball sampling method of church food pantries in Nashville, Tennessee, from 2015-2018. The study population included 93 urban churches with 46 having on-site food pantries.

Measurable Outcome/Analysis: Zip codes for churchbased pantries and clients served were collected via an electronic survey tool sent to urban churches. Social network ties between church food pantries and clients were analyzed utilizing UCINET 6 software.

Results: From the social network analysis a sociogram (eg visual matrix) was developed depicting 56 nodes (26 churches; 30 client zip codes) and 148 ties. Five large churches (membership $\geq 300$ ), 13 medium-sized churches (membership $=100$ to 299), and 8 small churches (membership $\leq 100$ ) were identified within 23 zip codes. 